\title{
Numerical Evaluation of Wiener Integrals
}

\author{
By Alan G. Konheim and Willard L. Miranker
}

Abstract. A systematic study of quadrature formulae for the Wiener integral $\int F[x] w(d x)$ of the type $\int F[\theta(u, \cdot)] \nu(d u)$ is presented. The Cameron and Vladimirov quadrature formulae, which are the function space analogues of Simpson's Rule, are shown to fit into this framework. Numerical results are included.

1. Introduction. In this paper we consider the problem of obtaining numerical approximations for the Wiener integral. The solution of the corresponding problem for multiple Riemann integrals is provided by a class of quadrature formulae of the type

$$
\iint \cdots \int f\left(x_{1}, x_{2}, \cdots, x_{n}\right) d x_{1} d x_{2} \cdots d x_{n} \cong \sum_{i=1}^{m_{n}} \lambda_{i, n} f\left(\mathbf{x}_{i, n}\right)
$$

where the constants $\left\{\lambda_{i, n}\right\}$ and points $\left\{\mathbf{x}_{i, n}\right\}$ are chosen by a variety of means. Since the Wiener integral of a functional $F$ may, in many instances, be defined as a suitable limit of repeated Riemann integrals, we are led to consider quadrature formulae, which are formal limits of (1.1) of the form

$$
\int_{C} F[x] w(d x) \cong \int F[\theta(u, \cdot)]_{\nu}(d u) .
$$

Here, $\int_{C} F[x] w(d x)$ denotes the Wiener integral, and $\int F[\theta(u, \cdot)] \nu(d u)$ denotes an integral over some Euclidean space.

In [1] Cameron determined a pair $(\nu, \theta)$ by imposing on (1.2) the condition that the formula be exact for polynomial functionals of degree $\leqq 3$. Imposing the same requirement, Vladimirov [5] constructed a family of pairs $(\nu, \theta)$.

In this paper we shall develop a class of approximations of the type given in (1.2). The pair $(\nu, \theta)$ is chosen so that the resulting quadrature formula

$$
\int F[\theta(u, \cdot)] \nu(d u)
$$

is exact for polynomial functionals of degree $\leqq 2 n+1$. The Cameron and Vladimirov results are special cases.

Quadrature formulae for Riemann integrals achieve a certain order of accuracy by the imposition of a requirement of exactness for polynomials of degree $\leqq k$. To increase the accuracy the range of integration is partitioned into subsets of "fineness" $\Delta$, and the quadrature formula applied within each subset. Under certain conditions the approximation may then be shown to have an error $O\left(\Delta^{e(k)}\right)$ as $\Delta \rightarrow 0$ where the exponent $e(k)$ is characteristic of the quadrature procedure. The analogue of the partition of the range of integration is also available for Wiener integrals. It takes the form of integrating over finite dimensional subspaces of $C$ (the space of continuous functions on $[0,1]$ which vanish at 0$)$. Using this device Cameron obtained the error estimate $O\left(\mathrm{~m}^{-2}\right)$ while Vladimirov proved only the estimate

Received May 16, 1966. 
$o(1)$ where $m$ denotes the dimensionality of this subspace. This method, when applied to our quadrature formulae, yields error estimates of the form

$$
O\left(m^{-(n+1-\eta)}\right)(\text { or } \quad o(1))(\text { as } m \rightarrow \infty)
$$

where the exponent $\eta$ depends upon the pair $(\nu, \theta)$. We are thus able to provide an analogue of the classical quadrature theory for Riemann integrals.

The Wiener integral plays a role in such diverse fields as probability theory, differential equations, statistical mechanics, and quantum physics [3]. Extraction of numerical values from the solutions of problems which are expressed in terms of Wiener integrals is therefore of some interest. For example, in [2] Gelfand, Frolov, and Čencov evaluated function integrals by a Monte Carlo method after replacing the integral by an iterated Riemann integral of many hundredfold order.

In Section 2 we state several known properties of the Wiener integral which are required in the sequel. In Section 3 we formulate the problem for quadrature formulae which are exact for polynomial functionals of degree $2 n+1$. We then show in Theorem 1 how to reduce the development of these formulae to the case of $n=1$. In Section 4 we characterize the most general $L^{2}$ solution for the case $n=1$ and give three specific examples. The first two examples correspond, respectively, to the formulae of Cameron and Vladimirov. In Section 5 we describe the refinement procedure for the quadrature formulae which consists of integrating over subspaces. We then derive error estimates for the mixed integration procedures. In Section 6 we give a summary of numerical evaluations of the Wiener integral of two specific functionals. We also include for reference a table of constants essential for the method developed here.

2. Some Properties of the Wiener Integral. Let $(C, \mathfrak{e}, w)$ denote the probability space consisting of

(1) $C$, the space of real-valued continuous functions $x(t)$ on $0 \leqq t \leqq 1$ with $x(0)=0$.

(2) $\mathcal{e}$, the $\sigma$-field of subsets of $C$ generated by the cylinder sets

$$
\begin{aligned}
\gamma_{\mathbf{a}, \mathbf{b}, \mathbf{t}} & =\left\{x \in C ;-\infty<a_{i}<x\left(t_{i}\right) \leqq b_{i}<\infty, 1 \leqq i \leqq n\right\}, \\
\mathbf{a} & =\left(a_{1}, a_{2}, \cdots, a_{n}\right) \quad \mathbf{b}=\left(b_{1}, b_{2}, \cdots, b_{n}\right) \quad \mathbf{t}=\left(t_{1}, t_{2}, \cdots, t_{n}\right)
\end{aligned}
$$

and

(3) $w$, Wiener measure.*

A functional $F$ on $C$ is called a monomial of degree $m$ if

$$
F[x]=\int_{I^{m}} x\left(t_{1}\right) x\left(t_{2}\right) \cdots x\left(t_{m}\right) \mu_{m}\left(d t_{1} \times d t_{2} \times \cdots \times d t_{m}\right)
$$

where $I^{m}=[0,1] \times[0,1] \times \cdots \times[0,1](m$ copies $)$, and $\mu_{m}$ is a regular signed measure of finite total variation defined on the Borel sets of $I^{m}$. A functional $F$ is called $a$ polynomial of degree $k$ if it is a linear combination of monomials of degree $\leqq k$. We shall denote this latter class by $P_{k}$.

We now state three known lemmas which describe properties of Wiener integrals which will be needed below. Lemmas 1 and 3 give the value of Wiener in-

\footnotetext{
* We choose that Wiener measure with $\sigma=2^{-1 / 2}$; see [3] for precise notation and definition.
} 
tegrals of functionals of special types, while Lemma 2 is an identity giving the Wiener integral in termís of its values over subspaces.

Lemma 1 [4, p. 151]. If $f: R^{n} \rightarrow R^{1}$ is such that

$$
I=\int_{R^{n}} e_{n}\left(u_{1}, u_{2}, \cdots, u_{n}\right) f\left(\frac{u_{1}}{2 i_{1}-1}, \cdots, \frac{u_{n}}{2 i_{n}-1}\right) d u_{1} \cdots d u_{n}
$$

exists (as a Lebesgue integral), where $i_{1}, i_{2}, \cdots, i_{n}$ are distinct positive integers and

$$
e_{h}^{\prime}\left(u_{1}, u_{2}, \cdots, u_{n}\right)=\pi^{-n / 2} \exp \left(-\left(u_{1}^{2}+u_{2}^{2}+\cdots+u_{n}^{2}\right)\right)
$$

then

$$
I=\int_{c} F[x] w(d x)
$$

where

$$
F[x]=f\left(\int_{0}^{1} \alpha_{i_{1}}(t) x(t) d t, \int_{0}^{1} \alpha_{i_{2}}(t) x(t) d t, \cdots, \int_{0}^{1} \alpha_{i_{n}}(t) x(t) d t\right)
$$

and

$$
\alpha_{j}(t)=2^{1 / 2} \sin (2 j-1) \pi t / 2 .
$$

Lemma $2\left[1\right.$, p. 114]. If $F \in L^{1}(C, \mathfrak{e}, w)$, then

$$
\int_{C} F[x] w(d x)=\int_{R^{n}} e_{n}(\mathbf{u}) d \mathbf{u} \int_{C} F\left[x-P_{n} x+\Psi_{n}(\mathbf{u}, \cdot)\right] w(d x)
$$

where

$$
\begin{aligned}
\left(P_{n} x\right)(t) & =\sum_{j=1}^{n} \alpha_{j}(t) \int_{0}^{1} \alpha_{j}(s) x(s) d s \\
\Psi_{n}(\mathbf{u}, t) & =\sum_{j=1}^{n} \frac{2}{\pi} \frac{u_{j} \alpha_{j}(t)}{2 j-1}
\end{aligned}
$$

and $e_{n}$ and $\alpha_{j}$ are as in (2.1) and (2.2).

LEMMA 3 [3, p. 50]. If $F[x]=x\left(t_{1}\right) x\left(t_{2}\right) \cdots x\left(t_{m}\right)\left(0 \leqq t_{i} \leqq 1,1 \leqq i \leqq m\right)$, then

$$
\begin{aligned}
& \int_{C} F[x] w(d x)=B_{m}\left(t_{1}, t_{2}, \cdots, t_{m}\right) \\
& =0 \text {, } \\
& m \text { odd, } \\
& =2^{-m / 2} \sum^{*} \min \left(t_{i_{1}}, t_{i_{2}}\right) \min \left(t_{i_{3}}, t_{i_{1}}\right) \cdots \min \left(t_{i_{m-1}}, t_{i_{m}}\right), \quad m \text { even, }
\end{aligned}
$$

the summation $\left(^{*}\right)$ being carried out over all partitions of $\{1,2, \ldots, m\}$ into $m / 2$ subsets each of size two.

3. Determination of the Pairs $\left(\nu_{n}, \theta_{n}\right)$. We seek an approximation formula to the Wiener integral of the type

$$
\int F[x] w(d x) \cong \int F\left[\theta_{n}(u, \cdot)\right] \nu_{n}(d u) .
$$


Using Lemma 3 above and arguments similar to those used by Cameron [1], it may be seen that the requirement that (3.1) be exact for polynomial functionals $F \in \mathcal{P}_{2 n+1}$ is equivalent to the conditions

$$
\begin{aligned}
\int \prod_{j=1}^{m} \theta_{n}\left(u, t_{j}\right) \nu_{n}(d u) & =B_{m}\left(t_{1}, t_{2}, \cdots, t_{m}\right), & & 1 \leqq m \leqq 2 n+1, \\
& =1, & & m=0 .
\end{aligned}
$$

In this section we will show how to construct pairs $\left(\nu_{n}, \theta_{n}\right)$ which satisfy the integral relations (3.2) in terms of pairs $(\nu, \theta)$ corresponding to $n=1$.

Let $\lambda$ denote a symmetric probability measure on the Borel sets $\Theta^{1}$ of $R^{1}$; i.e., $\lambda(B)=\lambda(-B)$ where $B \in \mathbb{B}^{1}$ and $-B=\{-b: b \in B\}$. We let $B_{[0,1]}^{1}$ denote the $\sigma$-field of Borel subsets of $[0,1]$, and suppose $\rho(\xi, t): R^{1} \times I^{1} \rightarrow R^{1}$ satisfies

(i) $\quad \rho$ is bounded and $B^{1} \times \Theta_{[0,1]}^{1}$ is measurable;

(ii) $\quad \rho(\xi, t)=-\rho(-\xi, t)$;

$$
\int \rho(\xi, t) \rho(\xi, s) \lambda(d \xi)=1 / 2 \min (s, t) .
$$

(Here and henceforth we omit the range of integration if it is over the entire space.) For $k$ a positive integer, $\lambda_{k}$ will denote the product measure on the Borel sets $\mathbb{B}^{k}$ of $R^{k}$ induced by $\lambda$.

In what follows $n$ is an arbitrary but fixed positive integer, and $\nu_{n}=\lambda_{n}$. Let $K^{1}$ denote the field of complex numbers, and define $\theta_{n}: R^{n} \times I^{1} \rightarrow K^{1}$ by

$$
\theta_{n}(\mathfrak{u}, t)=c_{1}(n) \rho\left(u_{1}, t\right)+c_{2}(n) \rho\left(u_{2}, t\right)+\cdots+c_{n}(n) \rho\left(u_{n}, t\right)
$$

where the complex constants $\left\{c_{j}(n)\right\}$ are to be specified.

TheOREM 1. If the $\left\{c^{2}{ }_{j}(n)\right\}$ are the roots of the polynomial

$$
A_{n}(z)=z^{n}-\frac{z^{n-1}}{1 !}+\frac{z^{n-2}}{2 !}-\cdots+(-1)^{n} \frac{1}{n !},
$$

then (3.2) holds where $\left(\nu_{n}, \theta_{n}\right)$ is given by (3.3)-(3.6).

Proof. Part I. Let $U$ be a finite set. By an n-partition of $U$ we shall mean a partition of $U,\left\{U_{1}, U_{2}, \cdots, U_{r}\right\}$ into $r$ (say) disjoint, nonvoid sets for some $r$ with $1 \leqq r \leqq n$. Denote by $\Pi_{n}(U)$ the family of all $n$-partitions of $U$. We say that an $n$-partition $\left\{U_{1}, U_{2}, \cdots, U_{r}\right\}$ of $U$ is of type $\alpha=\left(\alpha_{1}, \alpha_{2}, \cdots\right)$ if $\alpha_{i}$ of the sets $\left\{U_{j}\right\}_{j=1}^{r}$ have $i$ elements, for each $i, 1 \leqq i<\infty$. An n-partition is of even type $\alpha$ if $\alpha_{2 i+1}=0(0 \leqq i<\infty)$. Let $J_{n}(U)$ (resp. $\left.J_{n}{ }^{e}(U)\right)$ denote the totality of possible types (resp. even types) of $n$-partitions of $U$. Note that $\alpha \in J_{n}(U)$ if and only if

(i) $\alpha_{j}$ is a nonnegative integer $(1 \leqq j<\infty)$,

(ii) $\alpha_{1}+\alpha_{2}+\cdots \leqq n$,

(iii) $\alpha_{1}+2 \alpha_{2}+\cdots=|U|=$ number of elements in $U$.

Let $\prod_{n, \boldsymbol{\alpha}}(U)$ denote the family of $n$-partitions of $U$ of type $\alpha$.

We begin our proof by observing that, independent of the choice of the $\left\{c_{j}(n)\right\}$,

$$
\int \prod_{j=1}^{2 p+1} \theta_{n}\left(\mathfrak{u}, t_{j}\right) \nu_{n}(d \mathfrak{u})=B_{2 p+1}\left(t_{1}, t_{2}, \cdots, t_{2 p+1}\right)=0
$$

for all $p, 0 \leqq p<\infty$ due to the symmetries of $\lambda$ and $\rho$. It thus suffices to choose 
the $\left\{c_{j}(n)\right\}$ so that

$$
\int \prod_{j=1}^{2 p} \theta_{n}(\mathbf{u}, t) \nu_{n}(d \mathbf{u})=B_{2 p}\left(t_{1}, t_{2}, \cdots, t_{2 p}\right), \quad 1 \leqq p \leqq 2 n .
$$

Now,

$$
\prod_{j=1}^{2 p} \theta_{n}\left(\mathbf{u}, t_{j}\right)
$$

is a linear combination of terms of the form

$$
\prod_{k=1}^{r}\left(c_{i_{k}}(n)\right)^{\left|U_{k}\right|} \prod_{j \in U_{k}} \rho\left(x_{i_{k}}, t_{j}\right)
$$

with the expression in (3.8) corresponding to the $n$-partition $\left\{U_{1}, U_{2}, \cdots, U_{r}\right\}$ of $Z_{2 p}=\{1,2, \cdots, 2 p\}$. If we let

$$
E(U)=\int \prod_{j \in U} \rho\left(x, t_{j}\right) \lambda(d x),
$$

then the integral of the term in (3.8) with respect to the measure $\nu_{n}$ is equal to

$$
\prod_{k=1}^{r}\left(c_{i_{k}}(n)\right)^{\left|U_{k}\right|} E\left(U_{k}\right)
$$

To evaluate

$$
\int \prod_{j=1}^{2 p} \theta_{n}\left(\mathbf{u}, t_{j}\right) \nu_{n}(d \mathbf{u})
$$

we fix $\alpha \in{J_{n}}^{e}\left(Z_{2 p}\right),\left\{U_{1}, U_{2}, \cdots, U_{r}\right\} \in \prod_{n, \alpha}\left(Z_{2 p}\right)$ and sum (3.9) over all ordered $r$-tuples $\left(i_{1}, i_{2}, \cdots, i_{r}\right)$. There results

$$
\begin{aligned}
\int \prod_{j=1}^{2 p} \theta_{n}\left(\mathbf{u}, t_{j}\right) \nu_{n}(d \mathbf{u})= & \sum_{\alpha \in J_{n}^{e}\left(z_{2 p}\right)} \sum_{\left\{U_{1}, U_{2}, \cdots, U_{r}\right\} \in \mathbf{U}_{n, \boldsymbol{\alpha}}\left(z_{2_{p}}\right)} \\
& \left\{\prod_{j=1}^{r} E\left(U_{j}\right)\right\}\left\{\sum_{\left(i_{1}, i_{2}, \cdots, i_{r}\right)}^{*} \prod_{k=1}^{r}\left(c_{i_{b}}(n)\right)^{\left|U_{k}\right|}\right\}
\end{aligned}
$$

where the ${ }^{*}$-summation is carried out over all ordered $r$-tuples $\left(i_{1}, i_{2}, \cdots, i_{r}\right)$ of distinct integers with $i_{j} \in Z_{n}(1 \leqq j \leqq n)$. We note that the right-hand side of (3.10) includes the expression

$$
\left\{\sum_{\left(i_{1}, i_{2}, \cdots, i_{p}\right)}^{*} \prod_{k=1}^{p}\left(c_{i_{k}}(n)\right)^{2}\right\} B_{2 p}\left(t_{1}, t_{2}, \cdots, t_{2_{p}}\right)
$$

which corresponds to the sum

$$
\sum_{\left.\mid U_{1}, U_{2}, \cdots, v_{p}\right\} \in \Pi_{n, \alpha}\left(z_{2 p}\right)}\left\{\prod_{j=1}^{p} E\left(U_{j}\right)\right\}\left\{\sum_{\left(i_{1}, i_{2}, \cdots, i_{p}\right)}^{*} \prod_{k=1}^{p}\left(c_{i_{k}}(n)\right)^{2}\right\}
$$

with $\alpha=(0, p, 0,0, \cdots)$. (3.5) shows that this is the case since for

$$
\boldsymbol{\alpha}=(0, p, 0,0, \cdots),
$$

the corresponding sets in the partition have two elements. The proof consists of 
showing that it is possible to choose the $\left\{c_{j}(n)\right\}$ so that the coefficient of

$$
B_{2 p}\left(t_{1}, t_{2}, \cdots, t_{2 p}\right)
$$

in (3.11) is equal to unity, while simultaneously forcing all of the remaining expressions in (3.10) to vanish (for each $p, 1 \leqq p \leqq n$ ). We introduce the coefficient functions $G_{r}\left(p ; v_{1}, v_{2}, \cdots, v_{r}\right)$ defined by

$$
G_{r}\left(p ; v_{1}, v_{2}, \cdots, v_{r}\right)=\sum_{\left(i_{1}, i_{2}, \cdots, i_{r}\right)}^{*} \prod_{j=1}^{r}\left(c_{i_{j}}(n)\right)^{2 v_{j}}
$$

where the $\left\{v_{j}\right\}$ are positive integers with sum $p$.

Lemma 4. The family of functions $\left\{G_{r}\left(p ; v_{1}, v_{2}, \cdots, v_{r}\right)\right\}$ satisfies

(i) $G_{r}\left(p ; v_{1}, v_{2}, \cdots, v_{r}\right)=G_{r}\left(p ; v_{\sigma(1)}, v_{\sigma(2)}, \cdots, v_{\sigma(r)}\right)$ where $\sigma$ is a permutation of $Z_{r}$,

$$
\begin{aligned}
& G_{r}\left(p ; v_{1}, v_{2}, \cdots, v_{r}\right)=G_{1}\left(v_{r} ; v_{r}\right) G_{r-1}\left(p-v_{r} ; v_{1}, v_{2}, \cdots, v_{r-1}\right) \\
&-\sum_{i=1}^{r-1} G_{r-1}\left(p ; v_{1}, v_{2}, \cdots, v_{i-1}, v_{i}+v_{r}, v_{i+1}, \cdots, v_{r-1}\right)
\end{aligned}
$$

$f_{\text {or } r}>1$, and

$$
G_{r}\left(p ; v_{1}, v_{2}, \cdots, v_{r}\right)=\sum_{\beta \in \mathcal{J}_{r}\left(z_{r}\right)} C_{r}(\beta)
$$

$$
\left\{\sum_{\left\{N_{1}, \cdots, N_{s}\right\} \in \Pi_{r,}\left(Z_{r}\right)} \prod_{k=1}^{s} G_{1}\left(\sum_{j \in N_{k}} v_{j} ; \sum_{j \in N_{k}} v_{j}\right)\right\}
$$

where

$$
c_{r}(\boldsymbol{\beta})=\prod_{j=1}^{r}\left\{(-1)^{j-1}(j-1) !\right\}^{\beta_{j}} .
$$

Proof. Lemma 4 (i) is obvious. To prove Lemma 4 (ii) we need only observe that

$$
\begin{aligned}
G_{r}\left(p ; v_{1}, v_{2}, \cdots, v_{r}\right)= & \sum_{\left(i_{1}, i_{2}, \cdots, i_{r-1}\right)}^{*} \prod_{j=1}^{r-1}\left(c_{i_{j}}(n)\right)^{2 v_{j}} \\
& \times\left\{G_{1}\left(v_{r} ; v_{r}\right)-\left(c_{i_{1}}(n)\right)^{2 v_{r}}-\cdots-\left(c_{i_{r-1}}(n)\right)^{2 v_{r}}\right\} .
\end{aligned}
$$

The proof of Lemma 4 (iii) is by induction on $r$. Certainly, the result holds for $r=1$. Assuming the result for $r=t$, we then have by Lemma 4 (ii)

where

$$
\begin{aligned}
& G_{t+1}\left(p ; v_{1}, v_{2}, \cdots v_{t+1}\right)=G_{1}\left(v_{t+1} ; v_{t+1}\right) \\
& \times \sum_{\beta \in \mathfrak{J}_{t}\left(z_{t}\right)} C_{t}(\beta) \sum_{\left\{N_{1}, \cdots, N_{s}\right\} \in \Pi_{t, \beta}\left(z_{t}\right)} \prod_{k=1}^{s} G_{1}\left(\sum_{j \in N_{k}} v_{j} ; \sum_{j \in N_{k}} v_{j}\right) \\
& \quad-\sum_{i=1}^{t} \sum_{\beta \in \mathfrak{J}_{t}\left(z_{t}\right)} C_{t}(\beta) \sum_{\left\{N_{1}, \cdots, N_{s}\right\} \in \Pi_{t, \beta}\left(z_{t}\right)} \prod_{k=1}^{s} G_{1}\left(\sum_{j \in N_{k}} v_{j}{ }_{j}^{(i)} ; \sum_{j \in N_{k}} v_{j}{ }^{(i)}\right)
\end{aligned}
$$

$$
\begin{aligned}
& v_{j}^{(i)}=v_{j}, \quad \text { if } \quad j \neq i, j \in Z_{t}, \\
& =v_{i}+v_{t+1}, \quad \text { if } \quad j=i .
\end{aligned}
$$

Take $\gamma=\left(\gamma_{1}, \gamma_{2}, \cdots, \gamma_{t+1}, \cdots\right) \in J_{t+1}\left(Z_{t+1}\right)$; then (3.12) implies the recurrence 
formula

$$
\begin{aligned}
C_{t+1}(\gamma) & =C_{t}\left(\left(\gamma_{1}-1, \gamma_{2}, \cdots, \gamma_{t}, \cdots\right)\right) \quad \text { if } \gamma_{1} \geqq 1, \\
& =-(j-1) C_{t}\left(\left(0,0, \cdots, 0,1, \gamma_{j}-1, \gamma_{j+1}, \cdots, \gamma_{t+1}, \cdots\right)\right)
\end{aligned}
$$

if for some $j,(2 \leqq j \leqq t+1)$ we have $\gamma_{i}=0(1 \leqq i<j)$ and $\gamma_{j} \neq 0$.

Finally, (3.15) yields (3.12)-(3.13) for $i=t+1$. Note that $C_{t}(\boldsymbol{\gamma}) \neq 0$.

Proof. Part II. By virtue of (3.10)-(3.11) it suffices to choose the $\left\{c_{j}(n)\right\}$ so that

$$
\begin{aligned}
& G_{r}\left(p ; v_{1}, v_{2}, \cdots, v_{r}\right) \\
&=1 \quad \text { if } p=r \text { and } v_{1}=v_{2}=\cdots=v_{r}=1, \quad 1 \leqq p \leqq n . \\
&=0 \text { otherwise, }
\end{aligned}
$$

We assert that in order for (3.16) to hold it suffices $\dagger$ to require

$$
\begin{aligned}
G_{1}(p ; p)=1 & \text { if } \quad p=1, \\
=0 & \text { if } \quad 1<p \leqq n,
\end{aligned}
$$

For (3.17) implies that whenever $\left\{N_{1}, N_{2}, \cdots, N_{s}\right\} \in \prod_{r, \boldsymbol{\beta}}\left(Z_{r}\right),\left(\boldsymbol{\beta} \in \mathfrak{J}\left(Z_{r}\right)\right)$, then

$$
\begin{aligned}
\prod_{k=1}^{s} G_{1}\left(\sum_{j \in N_{k}} v_{j} ; \sum_{j \in N_{k}} v_{j}\right) & =0 \quad \text { if for any } k(1 \leqq k \leqq s) \sum_{j \in N_{k}} v_{j}>1 \\
& =1 \quad \text { if for every } k(1 \leqq k \leqq s) \sum_{j \in N_{k}} v_{j}=1
\end{aligned}
$$

which yields (3.16) by (3.12)-(3.13). Set $d_{j}(n)=c_{j}{ }^{2}(n)$ and let

$$
A_{n}(z)=\prod_{k=1}^{n}\left(z-d_{j}(n)\right)=\sum_{k=0}^{n} z^{n-k}(-1)^{k} \sigma_{k}(n)
$$

It is known [6, p. 81] that

$$
\sum_{j=0}^{p-1} G_{1}(p-j ; p-j)(-1)^{j} \sigma_{j}(n)+(-1)^{p} p \sigma_{p}(n)=0 \quad 1 \leqq p \leqq n
$$

which completes the proof of Theorem 1 by virtue of (3.17).

Remark. We assumed in (3.3) that $\rho$ is bounded. This requirement was made for purely technical reasons. Our conclusions are valid under the weaker condition

$$
\prod_{j=1}^{m} \rho\left(\cdot, t_{j}\right) \in L^{1}\left(R^{1}, \beta^{1}, \lambda\right), \quad 1 \leqq m \leqq 2 n+1 .
$$

4. The Solutions of $\int \rho(\xi, t) \rho(\xi, s) \lambda(d \xi)=\frac{1}{2} \min (s, t)$. In Section 3 we reduced the problem of determining quadrature formulas to finding a solution pair of the integral relation (3.2) for the case $n=1$. We will solve this problem by determining the solutions of

$$
\int_{-\infty}^{\infty} \rho(x, t) \rho(x, s) \lambda(d x)=\frac{1}{2} \min (s, t)
$$

† This condition is actually necessary and sufficient, but we only require its sufficiency. 
subject to the conditions

$\lambda$ is a symmetric probability measure on $B^{1}$,

$$
\begin{aligned}
& \rho(x, t)=-\rho(-x, t)(\lambda \text { a.e. }), \\
& \rho \in L^{2}\left(R^{1} \times I^{1} ; B^{1} \times B_{[0,1]}^{1} ; \lambda \times \mu\right)
\end{aligned}
$$

where $\mu$ denotes Lebesgue measure.

Note that it suffices to determine the solutions of

$$
\int_{0}^{\infty} \tilde{\rho}(x, t) \tilde{\rho}(x, s) \tilde{\lambda}(d x)=\frac{1}{4} \min (s, t)
$$

subject to the conditions

$\left(4.2^{\prime}\right) \quad \tilde{\lambda}$ is a measure on the Borel sets $B_{+}{ }^{1}$ of $R_{+}{ }^{1}=[0, \infty), \tilde{\lambda}\left(R_{+}{ }^{1}\right)=\frac{1}{2}$,

$\left(4.4^{\prime}\right) \quad \tilde{\rho} \in L^{2}\left(R_{+}{ }^{1} \times I^{1} ; B_{+}{ }^{1} \times B_{[0,1]}^{1} ; \tilde{\lambda} \times \mu\right)$.

The solutions of (4.1)-(4.4) are then obtained by taking $\lambda$ to be the even extension of $\tilde{\lambda}$ to $B^{1}$ and $\rho$ the odd extension (in $x$ ) of $\tilde{\rho}$ to $R^{1} \times I^{1}$.

Theorem 2 . The solutions of $\left(4.1^{\prime}\right),\left(4.2^{\prime}\right)$, and $\left(4.4^{\prime}\right)$ are given by

$$
\tilde{\rho}(x, t) \sim \sum_{j, k=1}^{\infty} \phi_{j}(t) \Psi_{k}(x)
$$

where

(i) the $\left\{\phi_{j}\right\}$ satisfy $\int_{0}^{1} \frac{1}{4} \min (s, t) \phi_{j}(t) d t=\mu_{j} \phi_{j}(s)$, where

$$
\mu_{j}=\frac{1}{4}\left(\frac{2 j-1}{2} \pi\right)^{-2}, \quad(1 \leqq j<\infty) \quad \text { and } \quad \int_{0}^{1} \phi_{j}{ }^{2}(t) d t=1
$$

(ii) $\left\{\Psi_{k}\right\}$ is any complete orthonormal set in $L^{2}\left({R_{+}}^{1}, \Theta_{+}{ }^{1}, \tilde{\lambda}\right)$; and

(iii) $\left(\mathbf{a}_{j_{1}}, \mathbf{a}_{j_{2}}\right) \equiv \sum_{k=1}^{\infty} a_{j_{1}, k} a_{j_{2}, k}=\mu_{j_{1}} \delta_{j_{1}, j_{2}}$, where $\delta_{j_{1}, j_{2}}$ is the usual Kromecker delta.

Proof. It is known that

$$
\frac{1}{4} \min (s, t)=\sum_{j=1}^{\infty} \mu_{j} \phi_{j}(s) \phi_{j}(t) .
$$

Expanding $\tilde{\rho}$ in its Fourier series (4.5), we substitute into (4.1') and use Parseval's theorem and (4.6) to infer that

Since

$$
\left(a_{j_{1}}, a_{j_{2}}\right)=\mu_{j_{1}} \delta_{j_{1}, j_{2}}
$$

$$
\sum_{j=1}^{\infty} \mu_{j}<\infty
$$

our conclusion is obtained.

Example 1 (Cameron). Let $\lambda$ be Lebesgue measure on $\left[-\frac{1}{2}, \frac{1}{2}\right]$ and

$$
\begin{aligned}
\rho(u, t) & =1 / \sqrt{2} & & \text { if } 0<u \leqq \frac{1}{2} \text { and } 2 u \leqq t \leqq 1 \\
& =-\rho(-u, t) & & \text { if }-\frac{1}{2} \leqq u<0 \text { and }-2 u \leqq t \leqq 1 \\
& =0 & & \text { otherwise. }
\end{aligned}
$$


Example 2 (Vladimirov). Let $\lambda$ be the measure on $\mathbb{B}^{1}$ determined by

$$
\lambda\{(-\infty, x]\}=\sum_{-\infty<k<\infty ; k \leqq x} \lambda_{k}
$$

where

$$
\begin{aligned}
\lambda_{k} & =\frac{1}{2} \frac{1-\lambda_{0}}{\left(\frac{2 k-1}{2} \pi\right)^{2}} & & \text { if } 1 \leqq k<\infty \\
& =\lambda_{-k} & & \text { if }-\infty<k \leqq-1 \\
& =\lambda_{0} \in[0,1) & & \text { if } k=0
\end{aligned}
$$

and

$$
\begin{aligned}
\rho(k, t) & =\frac{1}{2}\left\{\frac{2}{1-\lambda_{0}}\right\}^{1 / 2} \sin (2 k-1) \frac{\pi}{2} t & & \text { if } 1 \leqq k<\infty \\
& =-\rho(-k, t) & & \text { if }-\infty<k \leqq-1 \\
& =0 & & \text { if } k=0 .
\end{aligned}
$$

Example 3. Starting from the formula

$$
\frac{1}{\pi} \int_{-\infty}^{\infty} \frac{\sin m x}{x} d x=\operatorname{sgn} m
$$

we observe

$$
\frac{1}{\pi} \int_{-\infty}^{\infty} \frac{1-\cos A x}{x} \frac{1-\cos B x}{x} d x=\min (A, B) \quad \text { if } \quad A, B \geqq 0 .
$$

We now define the pair $\left(\lambda^{(r)}, \rho^{(r)}\right)$ for $0<r<\infty$ by

$$
\begin{array}{rlrl}
\lambda^{(r)}(B) & =\int_{B \cap[-1,1]} \frac{3}{4} u^{2} d u+\int_{B \cap[-1,1] c} \frac{2 r+1}{4} \frac{d u}{|u|^{2 r+2}} \\
\rho^{(r)}(u, t) & =\left(\frac{2}{3 \pi}\right)^{1 / 2} \frac{1-\cos t u}{u^{2}} & & \text { if } 0<u \leqq 1 \\
& =\left(\frac{2}{(2 r+1) \pi}\right)^{1 / 2}|u|^{r}(1-\cos t u) & & \text { if } 1<u<\infty \\
& =-\rho^{(r)}(-u, t) & & \text { if }-\infty<u<0 .
\end{array}
$$

Let $\mho_{n}$ denote the total variation of $\theta_{n}$ with respect to $t$ on $0 \leqq t \leqq 1$. Then,

\begin{tabular}{c|l|l|ll}
\hline \multicolumn{2}{c}{ Example 1 } & \multicolumn{2}{c}{ Example 2 } & \multicolumn{2}{c}{ Example 3 } \\
\hline$\theta_{n}$ & $\in L^{\infty}\left(R^{1} \times I^{1}\right)$ & $\in L^{\infty}\left(R^{1} \times I^{1}\right)$ & $\begin{array}{l}\in L^{2+(1 / r)-\eta}\left(R^{1} \times I^{1}\right) \\
\notin L^{2+(1 / r)}\left(R^{1} \times I^{1}\right)\end{array}$ & for all $\eta>0$ \\
\hline$v \theta_{n}$ & $\in L^{\infty}\left(R^{1} ; \nu_{n}\right)$ & $\notin L^{1}\left(R^{1} ; \nu_{n}\right)$ & $\begin{array}{l}\in L^{1+(r / r+1)-\eta}\left(R^{1} ; \nu_{n}^{(r)}\right) \\
\notin L^{1+(r / r+1)}\left(R^{1} ; \nu_{n}^{(r)}\right)\end{array}$ & for all $\eta>0$ \\
\hline
\end{tabular}


5. The Error Estimate. Suppose $\mathfrak{L}_{k}^{[a, b]}$ is a quadrature formula for the Riemann integral $\int_{a}^{b} f(x) d x$ chosen so that $\mathcal{L}_{k}^{[a, b]}(f)=\int_{a}^{b} f(x) d x$ whenever $f$ is a polynomial of degree $k$. In order to improve the accuracy of such a formula the interval of integration $[a, b]$ is partitioned into subintervals $\left\{\Delta_{i}\right\}_{i=1}^{m}$ and the formula applied within each subinterval; i.e., we approximate

$$
\int_{a}^{b} f(x) d x
$$

by

$$
\sum_{i=1}^{m} \mathfrak{L}_{k}^{\Delta_{i}}(f)
$$

Under suitable conditions the error

$$
\mathcal{E}(f)=\sum_{i=1}^{m} \mathfrak{L}_{k}^{\Delta_{i}}(f)-\int_{a}^{b} f(x) d x
$$

is $O\left(\Delta^{e(k)}\right)$ as $\Delta=\max \left|\Delta_{i}\right| \rightarrow 0$, the exponent $e(k)$ being characteristic of the quadrature formula $\mathcal{L}_{k}^{[a, b]}$. The subdivision of the range of integration is also at our disposal in the Wiener integral. The form it takes is given by Cameron's Mixed Integration Theorem (Lemma 2). The exponent $e(k)$, as we shall see, is dependent upon the integrability of $v \theta_{n}$.

Let $C^{*}$ denote the family of functions on $[0,1]$ of the form

$$
x(\cdot)+\left(I-P_{m}\right) \theta_{n}(\mathbf{u}, \cdot)
$$

where $x \in C, 1 \leqq m<\infty$ and $\mathbf{u} \in R^{n}$.

Theorem 3. Let $F \in L^{1}(C, \mathcal{e}, w)$ admit an extension to $C^{*}$ which we continue to denote by $F$. Suppose

$$
\begin{aligned}
F\left[x_{0}+x\right]=F\left[x_{0}\right]+\sum_{j=1}^{2 n+1} \int_{I} \prod_{k=1}^{j} x\left(t_{k}\right) \mu_{j, x_{0}}\left(d t_{1} \times \cdots\right. & \left.\times d t_{j}\right) \\
& +\mathbb{R}_{n}\left(x_{0} ; x\right) x_{0}, x \in C
\end{aligned}
$$

where

(i) $\mu_{j, x_{0}}$ is a regular signed measure of finite total variation, $\left\|\mu_{j, x_{0}}\right\|$, with

$$
\begin{gathered}
\left\|\mu_{j, x_{0}}\right\| \in L^{1}(C, \mathcal{e}, w), \\
\left|\mathcal{R}_{n}\left(x_{0} ; x\right)\right| \leqq\left\{\int_{0}^{1}|x(s)|^{2} d s\right\}^{n+1} \\
\times\left\{A_{1} \exp B \int_{0}^{1}\left|x(s)+x_{0}(s)\right|^{2} d s+A_{2} \exp B \int_{0}^{1}\left|x_{0}(s)\right|^{2} d s\right\}, \\
x_{0}, x \in C ; 0<B<\pi^{2} / 4,
\end{gathered}
$$

and such that

(iii) equations (5.1)-(5.3) continue to hold for $x_{0} \in C$ and $x^{*} \in C^{*}$.

Then, if $\theta_{n}$ is bounded, we have, as $m \rightarrow \infty$, 


$$
\begin{aligned}
& \varepsilon_{m, n}(F)=\int_{R^{m}} e_{m}(\mathbf{y}) d \mathbf{y} \int_{C} F\left[\left(I-P_{m}\right) x+\Psi_{m}(\mathbf{y}, \cdot)\right] w(d x) \\
& -\int_{R^{m}} e_{m}(\mathbf{y}) d \mathbf{y} \int_{R^{n}} F\left[\left(I-P_{m}\right) \theta_{n}(\mathbf{u}, \cdot)+\Psi_{m}(\mathbf{y}, \cdot)\right] \nu_{n}(d \mathbf{u}) \\
& = \begin{cases}o(1) & \text { if } \mathcal{V} \theta_{n} \notin L^{p}\left(R^{n}, \mathbb{B}^{n}, \nu_{n}\right) \text { for any } p>1 \\
o\left(m^{-\eta}\right) & \text { if } \mathcal{V} \theta_{n} \in L^{p}\left(R^{n}, \mathbb{B}^{n}, \nu_{n}\right) \text { for some } p, \\
& 1<p<2 n+2 \\
& \text { where } \eta=\left[\frac{p}{2}\right]+\left(p-2\left[\frac{p}{2}\right]-1\right)^{+} \\
O\left(m^{-(n+1)}\right) & \text { if } \mathcal{V} \theta_{n} \in L^{2(n+1)}\left(R^{n}, \otimes^{n}, \nu_{n} .\right)\end{cases}
\end{aligned}
$$

where

(i) $\Psi_{m}(\mathrm{y}, t)=\sum_{j=1}^{m}(2 / \pi)\left(y_{j} /(2 j-1)\right) \sqrt{2} \sin (2 j-1) \pi t / 2$,

(ii) $\left.{ }^{*}\right]$ is the "integer part of ${ }^{*}$, , and

(iii) $a^{+}=\max (a, 0)$.

Proof. We have

$$
\begin{array}{r}
\mathcal{E}_{m, n}(F)=\mathcal{E}_{m, n, 1}(F)-\mathcal{E}_{m, n, 2}(F)=\int_{R^{m}} e_{m}(\mathbf{y}) d \mathbf{y} \int_{C} R_{n}\left(\Psi_{m}(\mathbf{y}, \cdot) ;\left(I-P_{m}\right) x\right) w(d x) \\
-\int_{R^{m}} e_{m}(\mathbf{y}) d \mathbf{y} \int_{R^{n}} R_{n}\left(\Psi_{m}(\mathbf{y}, \cdot) ;\left(I-P_{m}\right) \theta_{n}(\mathbf{u}, \cdot)\right) \nu_{n}(d \mathbf{u})
\end{array}
$$

by virtue of the construction of the pair $\left(\nu_{n}, \theta_{n}\right)$ and the fact that

$$
\sum_{j=1}^{2 n+1} \int_{I} \prod_{i=1}^{j}\left(\left(I-P_{m}\right) x\right)\left(t_{k}\right) \mu_{j, \Psi_{m}(\mathbf{y}, \cdot)}\left(d t_{1} \times \cdots \times d t_{j}\right)
$$

is an element of $\mathcal{P}_{2 n+1}$. We shall estimate $\mathcal{E}_{m, n, 1}(F)$ and $\mathcal{E}_{m, n, 2}(F)$ separately. Since

$$
\int_{0}^{1}\left(\left(I-P_{m}\right) x(s)\right)^{2} d s=\sum_{j>m} \frac{4}{\pi^{2}}\left\{\int_{0}^{1} x(s) \alpha_{j}(s) d s\right\}^{2},
$$

we have, by Lemma 1 ,

$$
\int_{C}\left\{\int_{0}^{1}\left(\left(I-P_{m}\right) x(s)\right)^{2} d s\right\}^{n+1} w(d x)=O\left(m^{-(n+1)}\right)
$$

as $m \rightarrow \infty$. Since

$$
\begin{aligned}
\exp B \int_{0}^{1} \mid \Psi_{m}(\mathbf{y}, s)+((I & \left.\left.-P_{m}\right) x(s)\right)\left.\right|^{2} d s \\
& =\exp B \int_{0}^{1} \Psi_{m}{ }^{2}(\mathbf{y}, s) d s \cdot \exp B \int_{0}^{1}\left(\left(I-P_{m}\right) x(s)\right)^{2} d s \\
& \leqq \exp B \int_{0}^{1} \Psi_{m}{ }^{2}(\mathbf{y}, s) d s \cdot \exp B \int_{0}^{1} x^{2}(s) d s,
\end{aligned}
$$

we have, by Hölder's inequality, 


$$
\begin{aligned}
& \int_{C}\left\{\int_{0}^{1}\left(\left(I-P_{m}\right) x(s)\right)^{2} d s\right\}^{n+1} \\
& \quad \times\left\{\exp B \int_{0}^{1}\left|\Psi_{m}(\mathrm{y}, s)+\left(\left(I-P_{m}\right) x(s)\right)\right|^{2} d s\right\} w(d x) \\
& =\exp B \int_{0}^{1}{\Psi_{m}}^{2}(\mathrm{y}, s) d s \times O\left(m^{-(n+1)}\right)
\end{aligned}
$$

Finally,

$$
\int_{R^{m}} e_{m}(\mathbf{y})\left\{\exp B \int_{0}^{1} \Psi_{m}^{2}(\mathbf{y}, s) d s\right\} d \mathbf{y} \rightarrow\left(\sec \left(B^{1 / 2}\right)\right)^{1 / 2} \quad \text { as } m \rightarrow \infty,
$$

which, with (5.5), shows that

$$
\varepsilon_{m, n, 1}(F)=O\left(m^{-(n+1)}\right) \quad \text { as } m \rightarrow \infty .
$$

In estimating the rate at which $\varepsilon_{m, n, 2}(F) \rightarrow 0$, we no longer have Lemma 1 at our disposal. Let us assume that $\theta_{n} \in L^{2 r+1+\epsilon}\left(R^{n}, \mathbb{B}^{n}, \nu_{n}\right)$ where $r$ is an integer, $0 \leqq r \leqq$ $n$ and $0<\epsilon<1$. We have

$$
\begin{aligned}
\int_{0}^{1}\left|\left(I-P_{m}\right) \theta_{n}(\mathbf{u}, s)\right|^{2} d s=\sum_{j>m} \frac{4}{\pi^{2}}\left|\int_{0}^{1} \alpha_{j}(s) \theta_{n}(\mathbf{u}, s) d s\right|^{2} \\
=\sum_{j>m} \frac{4}{\pi^{2}} \frac{2}{(2 j-1) \pi / 2}\left|\int_{0}^{1} \cos (2 j-1) \frac{\pi}{2} s d_{s} \theta_{n}(\mathbf{u}, s)\right|^{2} \\
=\left|\left(\mathcal{V} \theta_{n}\right)(\mathbf{u})\right|^{2} O\left(m^{-1}\right),
\end{aligned}
$$

which yields

$$
\left\{\int_{0}^{1}\left|\left(I-P_{m}\right) \theta_{n}(\mathbf{u}, s)\right|^{2} d s\right\}^{r}=\left|\left(\mathcal{V}_{n}\right)(\mathbf{u})\right|^{2 r} O\left(m^{-r}\right) .
$$

On the other hand,

$$
\int_{0}^{1}\left|\left(I-P_{m}\right) \theta_{n}(\mathbf{u}, s)\right|^{2} d s \leqq \sum_{j>m} \frac{4}{\pi^{2}}\left|\frac{2}{(2 j-1) \pi / 2}\right|^{1+\epsilon}
$$

$$
\begin{aligned}
& \times\left|\left(v \theta_{n}\right)(\mathbf{u})\right|^{1+\epsilon}\left|\int_{0}^{1} \theta_{n}(\mathbf{u}, s) \alpha_{j}(s) d s\right|^{1-\epsilon} \\
= & \left|\left(v \theta_{n}\right)(\mathbf{u})\right|^{1+\epsilon} o\left(m^{-\epsilon}\right) .
\end{aligned}
$$

Taken together, (5.8) and (5.9) imply

$$
\begin{gathered}
\int_{R^{n}}\left\{\int_{0}^{1}\left|\left(I-P_{m}\right) \theta_{n}(\mathbf{u}, s)\right|^{2} d s\right\}^{(n+1)} \nu_{n}(d \mathbf{u}) \\
=\int\left|\left(\mathcal{V} \theta_{n}\right)(\mathbf{u})\right|^{2 r+1+e} o\left(m^{-(r+\varepsilon)}\right) \nu_{n}(d \mathbf{u}) .
\end{gathered}
$$

We again note that 


$$
\begin{aligned}
\exp B \int_{0}^{1}\left|\Psi_{m}(\mathbf{y}, s)+\left(I-P_{m}\right) \theta_{n}(\mathbf{u}, s)\right|^{2} d s \\
\quad=\exp B \int_{0}^{1} \Psi_{m}{ }^{2}(\mathbf{y}, s) d s \cdot \exp B \int_{0}^{1}\left|\left(I-P_{m}\right) \theta_{n}(\mathbf{u}, s)\right|^{2} d s \\
\quad \leqq \exp B \int_{0}^{1} \Psi_{m}{ }^{2}(\mathbf{y}, s) d s \cdot \exp B \int_{0}^{1}\left|\theta_{n}(\mathbf{u}, s)\right|^{2} d s \\
\quad \leqq D \exp \int_{0}^{1} \Psi_{m}{ }^{2}(\mathbf{y}, s) d s
\end{aligned}
$$

since $\theta_{n}$ is bounded. Equations (5.10) and (5.11) together yield

$$
\mathcal{E}_{m, n, 2}(F)=o\left(m^{-\eta}\right)
$$

with $\eta=r+\epsilon$, and hence, $\mathcal{E}_{m, n}(F)=o\left(m^{-\eta}\right)$. If $v \theta_{n} \in L^{2(n+1)}\left(R^{n}, \mathbb{B}^{n}, \nu_{n}\right)$, then we may take $r=n+1$ in (5.8) and replace $\eta$ in (5.12) by $n+1$ and " $o$ " by “ $O$ ". Finally, if $v \theta_{n} \notin L^{p}\left(R^{n}, \mathrm{~B}^{n}, \nu_{n}\right)$ for any $p>1$, then Parseval's theorem, applied to the left member of $(5.10)$, yields $\mathcal{E}_{m, n, 2}(F)=o(1)$, and hence, $\mathcal{E}_{m, n}(F)=$ $o(1)$.

6. Numerical Results. In this section we will give a summary of the results of numerical experiments performed with certain of the formulas derived above. We evaluate the functionals by a variety of formulas. In most cases our formulas reduce to evaluating finite-fold integrals. We do this by Monte Carlo. The slowness of the convergence of the Monte Carlo method for integrals of large multiplicity is the limiting factor in accuracy of our results. We conclude this section with a table of the coefficients $c_{j}(n)$ needed in our methods.

The mixed integration formula developed in Section 5 is

$$
\int_{c} F[x] d_{w} x=\int_{R^{m}} e_{m}(y) d y \int_{c} F\left[\left(I-P_{m}\right) x+\Psi_{m}(y, \cdot)\right] w(d x) .
$$

We will first apply this formula to integrate the functional exp $\|x\|^{2}$. It is known that to five figures

$$
\int_{c} \exp \int_{0}^{1} x^{2}(t) d t w(d x)=1.3605 .
$$

We first use the approximation formulas corresponding to the Cameron type (Ex-

TABLE I (Eq. (6.3))

\begin{tabular}{r|c|c|c|c|c}
\hline \multirow{2}{*}{$m$} & \multicolumn{5}{|c|}{$n$} \\
\cline { 2 - 5 } & 2 & 3 & 4 & 5 & 6 \\
\hline 3 & 1.35 & 1.31 & 1.41 & 1.41 & \\
4 & 1.34 & 1.34 & 1.36 & 1.36 & 1.31 \\
10 & 1.39 & 1.34 & 1.39 & 1.43 & 1.38 \\
20 & 1.30 & 1.35 & 1.39 & 1.36 & 1.31 \\
30 & 1.39 & 1.31 & 1.33 & 1.38 & 1.40 \\
40 & 1.40 & 1.36 & 1.30 & & \\
50 & 1.33 & 1.32 & & &
\end{tabular}


ample 1 in Section 4) in the right member of (6.1). With $F[x]=\|x\|^{2}$ this becomes

$$
\begin{aligned}
\int_{R^{m}} e_{m}(y) d y \int_{-1 / 2}^{1 / 2} \cdots \int_{-1 / 2}^{1 / 2} \exp \left[\frac{4}{\pi^{2}} \sum_{j=m+1}^{\infty} \frac{1}{(2 j-1)^{2}}\right. \\
\times\left(\sum_{l=1}^{n} c_{l}(n) \operatorname{sgn} u_{l} \cos \left\{(2 j-1) \pi u_{l}\right\}\right)^{2} \\
\left.\quad+\frac{4}{\pi^{2}} \sum_{j=1}^{m_{2}} \frac{1}{(2 j-1)^{2}} y_{j}^{2}\right] d u_{1} \cdots d u_{n} .
\end{aligned}
$$

Here and hereafter, $n$ is the parameter describing the degree of exactness of the approximation formula.

The integration $d y$ in (6.3) may be performed to yield

$$
\begin{aligned}
\prod_{j=1}^{m}\left(1-\frac{4}{\pi^{2}(2 j-1)^{2}}\right)^{-1 / 2} \int_{-1 / 2}^{1 / 2} \cdots \int_{-1 / 2}^{1 / 2} \exp \left[\frac{4}{\pi^{2}} \sum_{j=m+1}^{\infty} \frac{1}{(2 j-1)^{2}}\right. \\
\left.\times\left(\sum_{l=1}^{n} c_{l}(n) \operatorname{sgn} u_{l} \cos \left\{(2 j-1) \pi u_{l}\right\}\right)^{2}\right] d u_{1} \cdots d u_{n} .
\end{aligned}
$$

The $m+n$-fold integral in (6.3) and the $m$-fold integral in (6.4) were evaluated by a Monte Carlo method. The results are given in Tables I and II below. In these tables we see that the results are in rather good agreement with the exact

\begin{tabular}{|c|c|c|c|c|}
\hline \multirow{2}{*}{$m$} & \multicolumn{4}{|c|}{$n$} \\
\hline & 2 & 3 & 4 & 5 \\
\hline $\begin{array}{r}3 \\
4 \\
10 \\
20 \\
30 \\
40 \\
50\end{array}$ & $\begin{array}{l}1.3567 \\
1.3570 \\
1.3576 \\
1.3585 \\
1.3589 \\
1.3592 \\
1.3594\end{array}$ & $\begin{array}{r}1.3554 \\
1.3572 \\
1.3578 \\
1.3586 \\
1.3589 \\
1.3592 \\
1.3593\end{array}$ & $\begin{array}{l}1.3571 \\
1.3567 \\
1.3579 \\
1.3585 \\
1.3589 \\
1.3592\end{array}$ & $\begin{array}{l}1.3554 \\
1.3573 \\
1.3576 \\
1.3585\end{array}$ \\
\hline \multicolumn{5}{|c|}{ TABLE III (Eq. (6.5)) $(n=2)$} \\
\hline \multicolumn{2}{|c|}{$m$} & & & \\
\hline & & & $\begin{array}{l}1.3264 \\
1.3263 \\
1.3263 \\
1.3270 \\
1.3308 \\
1.3314 \\
1.3317\end{array}$ & \\
\hline
\end{tabular}
answer 1.3605. Indeed, the error is at most 5\% in Table I while it is at most $0.22 \%$ in Table II. In Table II we see that the results are invariant with increasing $n$ but improve as expected with increasing $m$. On the other hand, Table I shows in-

TABLE II (Eq. (6.4)) 
sensitivity to both $m$ and $n$. Unfortunately, the quality of our results is limited by the necessity to use Monte Carlo methods for evaluating $m+n$ - or $n$-fold integrals. Tables I and II represent calculations which sample the integrands in (6.3) and (6.4) at 300 points. In Table II the relatively small multiplicity of integration enabled 300 samplings to produce excellent results. In Table I the Monte Carlo method needs very many more iterations before an accurate test of the quality of the methods can be made. Note, however, that the rows $m=3$ and $m=4$ in Table I (small multiplicity of integration) show the right relative behavior.

We next consider the approximation formula of the Vladimirov type (Example 2 in Section 4) applied to the right member of (6.1) with $F[x]=\exp \|x\|^{2}$. In this case all integrations may be performed, and we get for the approximation

$$
\begin{aligned}
2^{-m} \prod_{j=1}^{m}\left[(2 j-1)^{2} \pi^{2}-4\right]^{1 / 2} \sum_{k_{1} \cdots k_{n}} \lambda_{k_{1}} \cdots \lambda_{k_{n}} \\
\times \exp \left[\frac{1}{2} \sum_{\alpha, \beta=1}^{n} c_{\alpha}(n) c_{\beta}(n) \delta_{\left|k_{\alpha}\right|,\left|k_{\beta}\right|}\right. \\
\left.\times \operatorname{sgn}\left(k_{\alpha} k_{\beta}\right)\left(1-\sum_{j=1}^{m} \delta_{\left|k_{\alpha}\right|, j}\right)\left(1-\sum_{j=1}^{m} \delta_{\left|k_{\beta}\right|, 0}\right)\right] .
\end{aligned}
$$

This expression was evaluated for $n=2$ and several values of $m$. The results are given in Table III. The results have the proper behavior relative to $m$, and we see that, as expected, they are less accurate than those in Table II.

Now we consider the Wiener integral

$$
\int_{c} \exp -\left(\int_{0}^{1} V(x(\tau)) d \tau\right) w(d x)
$$

TABLE IV

\begin{tabular}{r|r|r|r|r}
\hline & \multicolumn{3}{|c}{$n$} \\
\cline { 2 - 4 } & 2 & 3 & 4 & 5 \\
\hline 3 & .64 & .64 & .66 & .66 \\
4 & .65 & .66 & .67 & .64 \\
5 & .66 & .62 & .64 & .69 \\
10 & .64 & .65 & .65 & .64 \\
20 & .65 & .66 & .65 & .65 \\
\hline
\end{tabular}

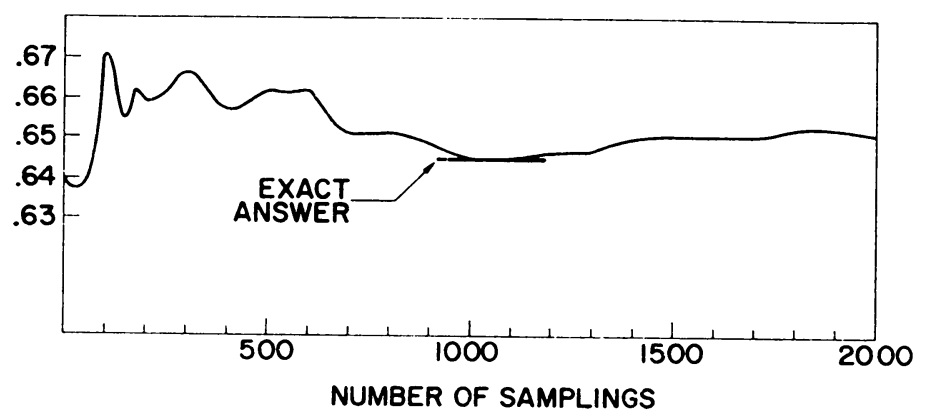

FIG. 1 
where

$$
\begin{array}{rlrl}
V(\xi) & =1 & & \xi>0 \\
& =0 & \xi \leqq 0 .
\end{array}
$$

This integral is the expected value of the fundamental solution at $t=1$ of the partial differential equation

$$
\frac{\partial u}{\partial t}=\frac{1}{2} \frac{\partial^{2} u}{\partial x^{2}}-V(x) u
$$

\section{TABle V}

$$
\begin{aligned}
& c_{1}(2)=.77688698-.32179713 i \\
& c_{2}(2)=.77688698+.32179713 i \\
& c_{1}(3)=.79154171 \\
& c_{2}(3)=.59266106+.40560610 i \\
& c_{3}(3)=.59266106-.40560610 i \\
& c_{1}(4)=.69700524+.16865045 i \\
& c_{2}(4)=.69700524-.16865045 i \\
& c_{3}(4)=.46880439+.42089298 i \\
& c_{4}(4)=.46880439-.42089298 i \\
& c_{1}(5)=.67719117 \\
& c_{2}(5)=.59917088+.25286159 i \\
& c_{3}(5)=.59917088-.25286159 i \\
& c_{4}(5)=.38364247+.41418658 i \\
& c_{5}(5)=.38364247-.41418658 i \\
& c_{1}(6)=.62251030+.10720664 i \\
& c_{2}(6)=.62251030-.10720664 i \\
& c_{3}(6)=.51649093+.29438975 i \\
& c_{4}(6)=.51649093-.29438975 i \\
& c_{5}(6)=.32260387+.40024508 i \\
& c_{6}(6)=.32260387-.40024508 i \\
& c_{1}(7)=.60203804 \\
& c_{2}(7)=.44947612+.31376828 i \\
& c_{3}(7)=.44947612-.31376828 i \\
& c_{4}(7)=.56252567+.17408303 i \\
& c_{5}(7)=.56252567-.17408303 i \\
& c_{6}(7)=.27715672+.38438162 i \\
& c_{7}(7)=.27715672-.38438162 i \\
& c_{1}(8)=.24221770+.36859030 i \\
& c_{2}(8)=.24221770-.36859030 i \\
& c_{3}(8)=.50659500+.21566945 i \\
& c_{4}(8)=.50659500-.21566945 i \\
& c_{5}(8)=.56540275+.07574939 i \\
& c_{6}(8)=.56540275-.07574939 i \\
& c_{7}(8)=.39536845+.32123876 i \\
& c_{8}(8)=.39536845-.32123876 i \\
&
\end{aligned}
$$


The exact value of this integral is known to be

$$
\frac{2}{\pi} \int_{0}^{\pi / 2} \exp \left(-\cos ^{2} \theta\right) d \theta=0.6450 .
$$

We use the mixed integration formula (6.1) and the approximations of the Cameron type. This leads to an $m+n$-fold integral which we do not reproduce here. This latter integral is evaluated by Monte Carlo. The result of sampling the integrand at 200 points is given in Table IV. The results in Table IV, with one exception, are within $4 \%$ of the exact answer. The deficiencies of Monte Carlo do not allow us to see the correct behavior of the results as $m$ and $n$ vary. To show the influence of the Monte Carlo method we ran the case $m=5, n=2$ in Table IV for 2000 samplings of the integrand. The final result for the integral was 0.652 . In Fig. 1 we reproduce a running account of this Monte Carlo calculation.

The integration formulas which we have derived require that $x(t)$ be replaced by a complex-valued expression since the $c_{j}(n)$ are complex-valued. If $F[x]$ is given, a priori, for real $x$ only as in (6.6) and (6.7), an appropriate complex extension must be chosen before our methods may be applied. In the function (6.7) we use as a complex extension

$$
\begin{aligned}
V(\xi) & =1 & & \operatorname{Re} \xi>0 \\
& =0 & & \operatorname{Re} \xi \leqq 0 .
\end{aligned}
$$

The method of prescribing a complex extension is not well defined. This matter remains an open question which we hope to look into.

Finally, we include in Table $\mathrm{V}$ a list of values of the coefficients $c_{j}(n)$ needed in our methods.

IBM Watson Research Center

Yorktown Heights, New York

1. R. H. Cameron, "A 'Simpson's Rule' for the numerical evaluation of Wiener's integrals in function space," Duke Math. J., v. 18, 1951, pp. 111-130. MR 12, 718.

2. I. M. Gelfand, A. S. Frolov \& N. N. Cencov, "The computation of continuous integrals by the Monte Carlo method," Izv. Vysš. Učebn. Zaved. Matematika, 1958, no. 5(6), pp. 32-45. (Russian) MR 24 \#B1739.

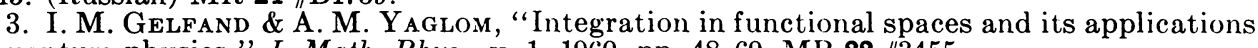
in quantum physics," J. Math. Phys., v. 1, 1960, pp. 48-69. MR 22 \#3455.

4. R. E. A. C. Paley \& N. Wiener, "Fourier transforms in the complex plane," Amer. Math. Soc. Colloq. Publ., Vol. 19, Amer. Math. Soc., Providence, R. I., 1934.

5. V. S. VLADIMIRov, "The approximate evaluation of Wiener integrals," Uspehi Mat. Nauk, v. 15, 1960, no. 4(94), pp. 129-135; English transl., Amer. Math. Soc. Transl. (2), v. 34, 1963, pp. 405-412. MR 23 \#A1404.

6. B. L. VAN DER WAERDEN, Moderne Algebra, Vol. I, Springer, Berlin, 1937; English transl., Ungar, New York, 1949-1950. MR 10, 587. 